\title{
High precision measurements of cross-sections in $A(d, p) X$ reactions at small internal proton momenta
}

\author{
Sergej Basilev ${ }^{a}$, Jurij Bushuev ${ }^{a}$, Viktor Glagolev ${ }^{a}$, Sergej Dolgiy ${ }^{a}$, Dmitrij Kirillov ${ }^{a}$, \\ Natalia Kostyaeva $^{a}$, Aleksandr Kovalenko $^{a}$, Aleksej Livanov $^{a}$, Petr Manyakov $^{a}$, \\ Gabriela Martinska $^{b}$, Jan Musinsky $^{c}$, Nikolaj Piskunov ${ }^{a}$, Anatolij Povtoreiko ${ }^{a}$, Pavel \\ Rukoyatkin $^{a}$, Roman Shindin ${ }^{a}$, Igor Sitnik*, Vjacheslav Slepnev $^{a}$, Il'ja Slepnev and \\ Jozef Urban ${ }^{b}$ \\ a Joint Institute for Nuclear Research, 141980 Dubna, Moscow region, Russia \\ ${ }^{b}$ University of P.J. Safarik, Jesenna. 5, SK-04154 Kosice, Slovak Republic \\ ${ }^{c}$ Institute of Experimental Physics, Watsonova 47, SK-04001 Kosice, Slovak Republic \\ E-mail: bsn6255amail.rul mone valagolevejinr.ru, mone \\ dkirillovdjinr.ru, nvkostiaevadgmail.ru, kovalendjinr.ru, \\ livanovdthe.jinr.ru, wowe, gabriela.martinskadupjs.sk \\ musinskydgmail.com, piskunovejinr.ru, aapovtoremail.ru, wome, \\ romanshindindyandex.ru, sitnikdabna.ru, vmslepnevegmail.com, \\ islepnevdgmail.com ozef.urbandupjs.sk
}

The deuteron breakup cross sections on $\mathrm{C}$ and $\mathrm{CH}_{2}$ targets are measured up to proton internal momenta of $0.3 \mathrm{GeV} / \mathrm{c}$. The obtained data are compared with previous measurements. The cross-sections ratio of $C(d, p) X$ and ${ }^{1} H(d, p) X$ reactions is obtained with high precision. The applying of the light front variable for the description of the breakup process is discussed. The measurements were performed at the Laboratory of High Energy Physics of Joint Institute for

Nuclear Research.

XXII International Baldin Seminar on High Energy Physics Problems,

15-20 September 2014

JINR, Dubna, Russia

\footnotetext{
${ }^{*}$ Speaker.
} 


\section{Introduction}

Disintegration of the lightest nuclei is one of effective tools in studying of the few-body problem. The first measurements of the $C(d, p) X$ in the entire range of the internal proton momenta were fulfilled in 1983 [四. The measurements were repeated with the higher precision on $C$ and $\mathrm{CH}_{2}$ targets in 1992 [回].

Here we present results of the new measurements on $\mathrm{C}$ and $\mathrm{CH}_{2}$ targets, which differ from the previous ones by:

- high resolution measurements of all momentum components of secondary protons;

- large angle-momentum acceptance for secondary protons;

- huge statistics within the proton internal momentum range of $0-0.3 \mathrm{GeV} / \mathrm{c}$.

\section{Experiment}

The setup "Strela" [B], shown in Fig.W, was situated on the extracted beam line of the Nuclotron. The primary deuteron beam with momentum of $3.5 \mathrm{GeV} / \mathrm{c}$ had the intensity $<10^{6}$ particles/sec, which made it possible to measure incident particle tracks. The bending angle in the magnet was of $0.289 \mathrm{rad}$. The scintillator counters were used for triggers. The track parameters were measured by drift chambers with the space accuracy about $0.05 \mathrm{~mm}$. The momentum resolution and efficiency of the setup were $\delta p / p \simeq 0.009$ and $\varepsilon=0.91 \pm 0.01$, respectively. The momentum acceptance of the setup is shown in Fig.】.

For the track reconstruction and search of the setup offsets the program package [四] was used.

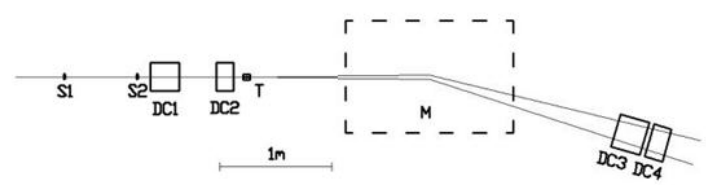

Figure 1: Main scheme of the experiment. $T$ - target, $\mathrm{M}-$ dipole, $S_{i}-$ scintillator counters, $D C_{i}-\mathrm{drift}$ chambers.

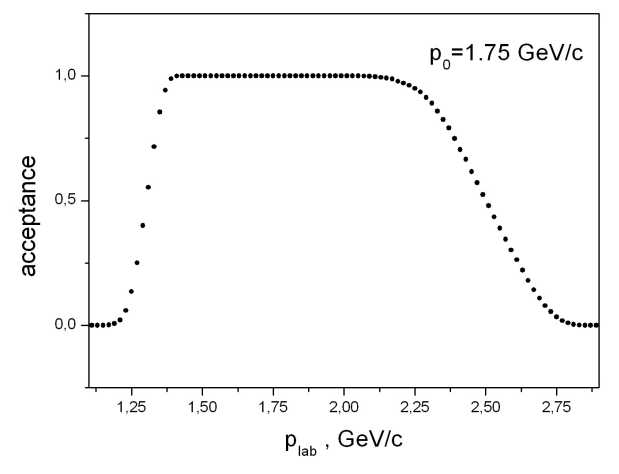

Figure 2: Geometric efficiency (acceptance) of the setup.

\section{Results}

For comparison between experiment and theory it is supposed that internal momentum of proton in deuteron is so called light front variable, $k$, firstly introduced by P.A.M. Dirac [[]]. Some 
theorists prefer to consider $q$, the proton momentum in the deuteron rest frame, as such. $k$ is related to $q$ as follows(z-axis is along beam line)[四:

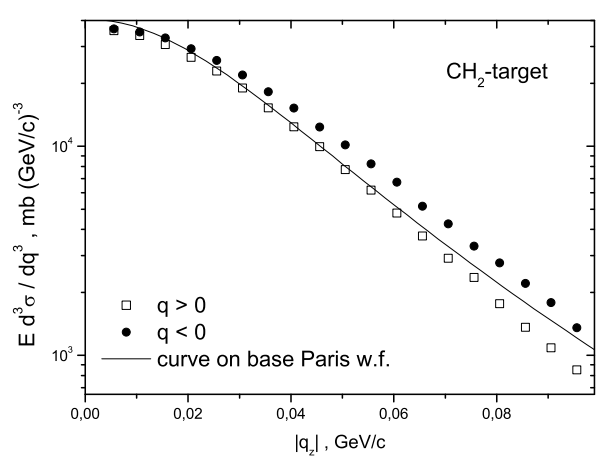

Figure 3: Comparison of the $\left.\operatorname{left}\left(q_{z}<0\right)\right)$ and $\operatorname{right}\left(q_{z}>0\right)$ slopes for invariant cross-sections.

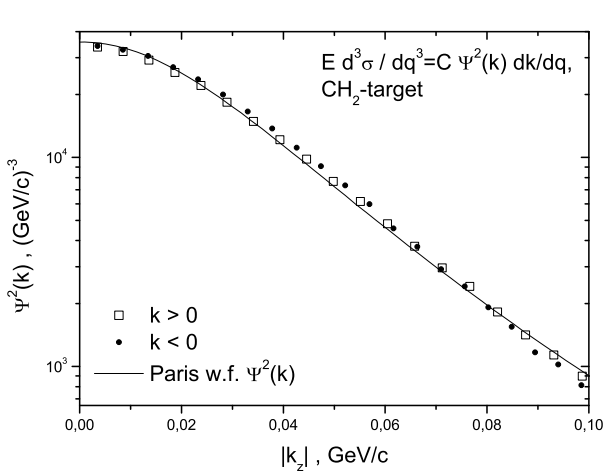

Figure 4: Comparison of the left and right slopes for $\Psi^{2}(k)$.

$$
\begin{aligned}
\alpha & =\frac{\sqrt{m_{s}^{2}+q^{2}}+q_{z}}{M} \\
M_{s f}^{2} & =\frac{q^{2}+m_{s}^{2}(1-\alpha)+m_{f}^{2} \alpha}{\alpha(1-\alpha)} \\
k_{z} & =\left(\alpha-\frac{1}{2}\right) M_{s f}-\frac{m_{s}^{2}-m_{f}^{2}}{2 M_{s f}} \\
k_{x, y} & =q_{x, y}
\end{aligned}
$$

where $m_{s}$ is the mass of the spectator (proton), $m_{f}$ is the mass of the second fragment, $\mathrm{M}$ is the deuteron mass. The obtained data are compared with theoretical predictions based on the Plane Wave Impulse Approximation. The advantage of $k$ in comparison with $q$ follows from comparison $C\left({ }^{3} \mathrm{He}, d\right) X$ and $C\left({ }^{3} \mathrm{He}, p\right) X$ spectra as functions of one or another variable[四]. But it does not mean that the "better" is completely correct. Therefore here is presented another approach to this problem. To provide condition

$$
q_{z} \simeq q\left(q \perp \ll q_{z}\right),
$$

events were selected with the restriction $q \perp<0.01 \mathrm{GeV} / \mathrm{c}$. Correctness of light front variable is demonstrated in Figs. [1, 4 . One can see that the invariant cross section has the distinctive asymmetry respectively the position of maximum, while $\Psi^{2}$-distribution is quite symmetrical. For the first time such an approach was applied in [8], but statistics was not too convincing.

In.Fig. [D the present data are compared with results of [D]. To provide condition (B.2), at $q_{z}<0.1 \mathrm{GeV} / \mathrm{c}$ the restriction on $q \perp$ was as mentioned above. At higher values of $q_{z}$, to provide acceptable statistics, the condition $q \perp<0.07 \mathrm{GeV} / \mathrm{c}$ was used.

The good agreement between two sets of data, including the region of the anomalous behavior of the cross sections in vicinity $0.3 \mathrm{GeV} / \mathrm{c}$, indicates the following:

- the authors of [Q], explaining the anomalous behavior of the cross sections, proceeded from the false assumption about the presence of systematic errors in data [0]; 
- the reaction mechanism, suggested to explain the same anomaly [ए]] has an energy dependence, while data do not confirm that.

In Fig.6 the ratio of differential cross-sections for $C(d, p) X$ and ${ }^{1} H(d, p) X$ reactions is demonstrated. The latter one was obtained by subtraction of $\mathrm{CH}_{2}$ and $\mathrm{C}$ cross-sections. To provide better statistics, events were collected, using the variable q':

$$
\begin{aligned}
& q^{\prime}=q=\sqrt{q_{z}^{2}+q \perp^{2}} \text { at } \quad q_{z}>0 \\
& q^{\prime}=-q \quad \text { at } \quad q_{z}<0
\end{aligned}
$$

Data show rather sharp peak at $|q|<0.05 \mathrm{GeV} /$ c. $q_{z}$-distribution without restriction on $q \perp$ agrees well with data [[] in the entire range of $q$. Ratio of maximum value and sloping part of the spectrum are close to predicted in [ए]].

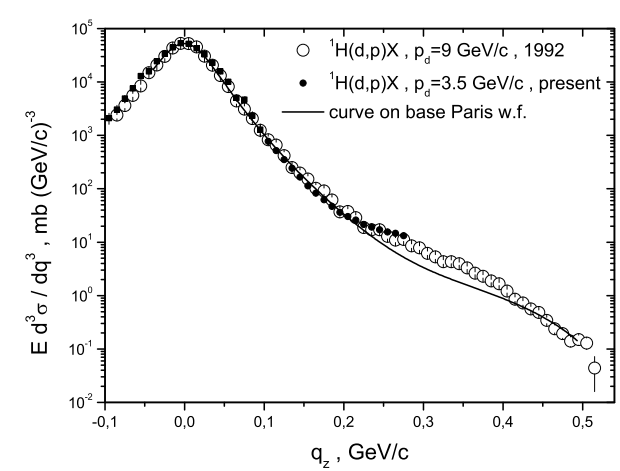

Figure 5: Invariant differential cross-section ${ }^{1} H(d, p) X$.

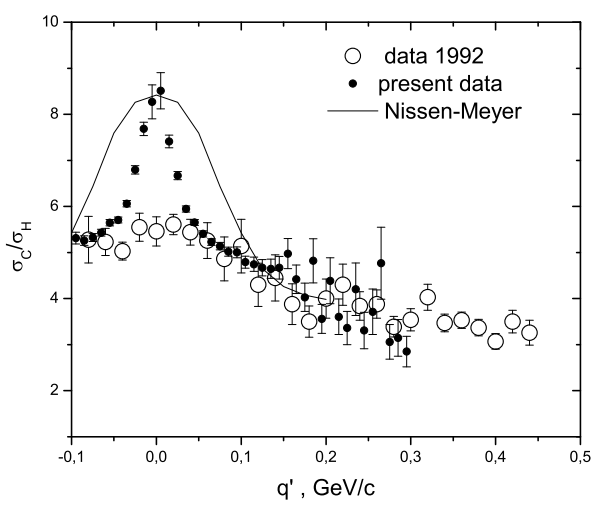

Figure 6: Cross-sections ratio of $C(d, p) X$ and ${ }^{1} H(d, p) X$ reactions.

\section{Conclusions}

- Light front variable is preferable for comparison experiment with theory in the entire range of the internal proton momenta.

- Breakup cross-sections show energy independence in $\mathrm{GeV}$ region.

- Ratio of breakup cross-sections on carbon and hydrogen demonstrates an interesting behavior in vicinity of zero internal momentum.

\section{References}

[1] V.G. Ableev et al. A Study of the Proton Momentum Spectrum From Deuteron Fragmentation at 8.9-GeV/c and an Estimate of Admixture Parameters for the Six Quark State in Deuteron, Nucl.Phys. A393 (1983) 491. 
[2] V.G. Ableev et al., Measurements of the ${ }^{12} C(d, p)$ and $p(d, p)$ forward cross-sections over a wide range of proton momenta, JINR Rapid. Com. 1[52]-92 (1992) 10.

[3] V.V. Glagolev et al., STRELA experimental setup for studying charge-exchange processes, Instr. and Experim. Techn., 56 (2013) 387.

[4] I.M. Sitnik, Development of the FUMILI minimization package, Comput.Phys.Commun. 185 (2014) 599; JINR Preprint E11-2010-101.

[5] P.A.M. Dirac, Forms of Relativistic Dynamics, Rev. Mod. Phys. 21 (1949) 392.

[6] V.A. Karmanov, Relativistic Composite Systems in the Light Front Dynamics (In Russian), Fiz.Elem.Chast.Atom.Yadra 19 (1988) 525.

[7] V.G. Ableev et al., Momentum Distribution of Protons and Deuterons From ${ }^{3}$ He Fragmentation at 10.78 GeV/c on Carbon at Zero Angles, JETP Lett. 45 (1987) 596.

[8] I.M. Sitnik, The Experimental test of the adequateness of relativistic impulse approximation when describing the lightest nuclei breakup, Phys. of Atomic Nuclei 59 (1996) 1059.

[9] L.S. Azhgirey, M.A. Ignatenko, N.P. Yudin, Possible interpretation of the "anomalous" behavior of the proton spectrum at $0^{\circ}$ from the deuteron fragmentation at $9 \mathrm{GeV} / \mathrm{c}$, Z.Phyz. A 343 (1992) 35.

[10] A.P. Ierusalimov, G.I. Lykasov, M. Viviani, Relativistic and spin effects in elastic backward p-d scattering, (2010) arXiv:1002.0249.

[11] S.Nissen-Meyer, A New Approximation for Glauber Theory on Stripping of Relativistic Deuterons, Nucl. Phys. A306 (1978) 499. 